\title{
Scalar mesons in a linear sigma model with (axial-)vector mesons
}

\author{
D. Parganlija*, P. Kovács ${ }^{\dagger}$, Gy. Wolf ${ }^{\dagger}$, F. Giacosa** and D.H. Rischke** \\ *Institute for Theoretical Physics, Vienna University of Technology, Wiedner Hauptstr. 8-10, \\ A-1040 Vienna, Austria \\ $\dagger$ Institute for Particle and Nuclear Physics, Wigner Research Center for Physics, Hungarian \\ Academy of Sciences, H-1525 Budapest, Hungary \\ ${ }^{* *}$ Institute for Theoretical Physics, Johann Wolfgang Goethe University, Max-von-Laue-Str. 1, \\ D-60438 Frankfurt am Main, Germany
}

\begin{abstract}
The structure of the scalar mesons has been a subject of debate for many decades. In this work we look for $\bar{q} q$ states among the physical resonances using an extended Linear Sigma Model that contains scalar, pseudoscalar, vector, and axial-vector mesons both in the non-strange and strange sectors. We perform global fits of meson masses, decay widths and amplitudes in order to ascertain whether the scalar $\bar{q} q$ states are below or above $1 \mathrm{GeV}$. We find the scalar states above $1 \mathrm{GeV}$ to be preferred as $\bar{q} q$ states.
\end{abstract}

Keywords: linear sigma model, scalar meson

PACS: $12.39 . \mathrm{Fe}, 12.40 . \mathrm{Yx}, 14.40 . \mathrm{Be}, 14.40 . \mathrm{Df}$

\section{INTRODUCTION}

Understanding the meson mass spectrum in the region below $2 \mathrm{GeV}$ is one of the fundamental problems of QCD. While the quark model seems to work very well for many resonances, some fundamental questions, such as the constituent quark content of scalar and axial-vector resonances, are still unanswered. The Particle Data Group (PDG) [1] suggests in the region below $1.8 \mathrm{GeV}$ the existence of five $I\left(J^{P C}\right)=0\left(0^{++}\right)$states: $f_{0}(500)$, $f_{0}(980), f_{0}(1370), f_{0}(1500)$, and $f_{0}(1710)$; two $I=1$ states: $a_{0}(980)$ and $a_{0}(1450)$; and two $I=1 / 2$ states: $K_{0}^{\star}(800)$ (or $\kappa$ ) and $K_{0}^{\star}(1430)$. A description of all mentioned scalar states as $\bar{q} q$ states is not possible, since the number of physical resonances is much larger than the number of resonances that can be constructed within a $\bar{q} q$ picture of mesons, e.g. in the isoscalar sector two states compared to five experimentally seen resonances. The question is: which of the five states are (predominantly) quarkonia?

Understanding these issues is not only crucial for hadron vacuum spectroscopy but is also important at nonzero temperatures and densities, because the correct identification of the chiral partner of the pion and of the $\rho$ meson is necessary for a proper description of the in-medium properties of hadrons [2]. The answers to this fundamental question is in principle contained in the QCD Lagrangian. Unfortunately, QCD cannot be solved by analytic means from first principles in the low-energy domain. For this reason, effective theories have been developed which share some of the underlying symmetries of QCD. The QCD Lagrangian exhibits, in addition to the local $S U(3)_{c}$ color symmetry and the discrete $C P T$ symmetry, a global $U\left(N_{f}\right)_{L} \times U\left(N_{f}\right)_{R} \equiv U(1)_{V} \times U(1)_{A} \times S U\left(N_{f}\right)_{V} \times$ $S U\left(N_{f}\right)_{A}$ chiral symmetry which is broken in several ways: spontaneously [due to the 
chiral condensate $\langle\bar{q} q\rangle=\left\langle\bar{q}_{R} q_{L}+\bar{q}_{L} q_{R}\right\rangle \neq 0$ ], explicitly (due to non-vanishing quark masses), as well as at the quantum level [the $U(1)_{A}$ anomaly].

In the framework of effective theories the chiral symmetry of QCD can be realized along two lines: linearly [3] and non-linearly [4]. In this contribution, we present a linear sigma model containing scalar, pseudoscalar, vector, and axial-vector mesons with both non-strange and strange quantum numbers. In view of the large number of the fields involved, our model shall be referred to as the "extended Linear Sigma Model", or "eLSM".

\section{THE MODEL}

The Lagrangian of our model [5] reads

$$
\begin{aligned}
\mathscr{L}= & \operatorname{Tr}\left[\left(D_{\mu} \Phi\right)^{\dagger}\left(D_{\mu} \Phi\right)\right]-m_{0}^{2} \operatorname{Tr}\left(\Phi^{\dagger} \Phi\right)-\lambda_{1}\left[\operatorname{Tr}\left(\Phi^{\dagger} \Phi\right)\right]^{2}-\lambda_{2} \operatorname{Tr}\left(\Phi^{\dagger} \Phi\right)^{2} \\
& -\frac{1}{4} \operatorname{Tr}\left(L_{\mu \nu}^{2}+R_{\mu \nu}^{2}\right)+\operatorname{Tr}\left[\left(\frac{m_{1}^{2}}{2}+\Delta\right)\left(L_{\mu}^{2}+R_{\mu}^{2}\right)\right]+\operatorname{Tr}\left[H\left(\Phi+\Phi^{\dagger}\right)\right] \\
& +c_{1}\left(\operatorname{det} \Phi-\operatorname{det} \Phi^{\dagger}\right)^{2}+i \frac{g_{2}}{2}\left(\operatorname{Tr}\left\{L_{\mu \nu}\left[L^{\mu}, L^{v}\right]\right\}+\operatorname{Tr}\left\{R_{\mu v}\left[R^{\mu}, R^{v}\right]\right\}\right) \\
& +\frac{h_{1}}{2} \operatorname{Tr}\left(\Phi^{\dagger} \Phi\right) \operatorname{Tr}\left(L_{\mu}^{2}+R_{\mu}^{2}\right)+h_{2} \operatorname{Tr}\left[\left(L_{\mu} \Phi\right)^{2}+\left(\Phi R_{\mu}\right)^{2}\right]+2 h_{3} \operatorname{Tr}\left(L_{\mu} \Phi R^{\mu} \Phi^{\dagger}\right) . \\
& +g_{3}\left[\operatorname{Tr}\left(L_{\mu} L_{v} L^{\mu} L^{v}\right)+\operatorname{Tr}\left(R_{\mu} R_{v} R^{\mu} R^{v}\right)\right]+g_{4}\left[\operatorname{Tr}\left(L_{\mu} L^{\mu} L_{v} L^{v}\right)+\operatorname{Tr}\left(R_{\mu} R^{\mu} R_{v} R^{v}\right)\right] \\
& +g_{5} \operatorname{Tr}\left(L_{\mu} L^{\mu}\right) \operatorname{Tr}\left(R_{v} R^{v}\right)+g_{6}\left[\operatorname{Tr}\left(L_{\mu} L^{\mu}\right) \operatorname{Tr}\left(L_{v} L^{v}\right)+\operatorname{Tr}\left(R_{\mu} R^{\mu}\right) \operatorname{Tr}\left(R_{v} R^{v}\right)\right], \quad(1)
\end{aligned}
$$

where

$$
\begin{aligned}
D^{\mu} \Phi & \equiv \partial^{\mu} \Phi-i g_{1}\left(L^{\mu} \Phi-\Phi R^{\mu}\right)-i e A^{\mu}\left[T_{3}, \Phi\right] \\
L^{\mu v} & \equiv \partial^{\mu} L^{v}-i e A^{\mu}\left[T_{3}, L^{v}\right]-\left\{\partial^{v} L^{\mu}-i e A^{v}\left[T_{3}, L^{\mu}\right]\right\} \\
R^{\mu v} & \equiv \partial^{\mu} R^{v}-i e A^{\mu}\left[T_{3}, R^{v}\right]-\left\{\partial^{v} R^{\mu}-i e A^{v}\left[T_{3}, R^{\mu}\right]\right\}
\end{aligned}
$$

The quantities $\Phi, R^{\mu}$, and $L^{\mu}$ represent the scalar and vector nonet:

$$
\begin{aligned}
& \Phi=\sum_{i=0}^{8}\left(S_{i}+i P_{i}\right) T_{i}=\frac{1}{\sqrt{2}}\left(\begin{array}{ccc}
\frac{\left(\sigma_{N}+a_{0}^{0}\right)+i\left(\eta_{N}+\pi^{0}\right)}{\sqrt{2}} & a_{0}^{+}+i \pi^{+} & K_{0}^{\star+}+i K^{+} \\
a_{0}^{-}+i \pi^{-} & \frac{\left(\sigma_{N}-a_{0}^{0}\right)+i\left(\eta_{N}-\pi^{0}\right)}{\sqrt{2}} & K_{0}^{\star 0}+i K^{0} \\
K_{0}^{\star-}+i K^{-} & \bar{K}_{0}^{\star 0}+i \bar{K}^{0} & \sigma_{S}+i \eta_{S}
\end{array}\right), \\
& L^{\mu}=\sum_{i=0}^{8}\left(V_{i}^{\mu}+A_{i}^{\mu}\right) T_{i}=\frac{1}{\sqrt{2}}\left(\begin{array}{ccc}
\frac{\omega_{N}+\rho^{0}}{\sqrt{2}}+\frac{f_{1 N}+a_{1}^{0}}{\sqrt{2}} & \rho^{+}+a_{1}^{+} & K^{\star+}+K_{1}^{+} \\
\rho^{-}+a_{1}^{-} & \frac{\omega_{N}-\rho^{0}}{\sqrt{2}}+\frac{f_{1 N}-a_{1}^{0}}{\sqrt{2}} & K^{\star 0}+K_{1}^{0} \\
K^{\star-}+K_{1}^{-} & \bar{K}^{\star 0}+\bar{K}_{1}^{0} & \omega_{S}+f_{1 S}
\end{array}\right)^{\mu}, \\
& R^{\mu}=\sum_{i=0}^{8}\left(V_{i}^{\mu}-A_{i}^{\mu}\right) T_{i}=\frac{1}{\sqrt{2}}\left(\begin{array}{ccc}
\frac{\omega_{N}+\rho^{0}}{\sqrt{2}}-\frac{f_{1 N}+a_{1}^{0}}{\sqrt{2}} & \rho^{+}-a_{1}^{+} & K^{\star+}-K_{1}^{+} \\
\rho^{-}-a_{1}^{-} & \frac{\omega_{N}-\rho^{0}}{\sqrt{2}}-\frac{f_{1 N}-a_{1}^{0}}{\sqrt{2}} & K^{\star 0}-K_{1}^{0} \\
K^{\star-}-K_{1}^{-} & \bar{K}^{\star 0}-\bar{K}_{1}^{0} & \omega_{S}-f_{1 S}
\end{array}\right)^{\mu},
\end{aligned}
$$


where the assignment to physical particles is shown as well ${ }^{1}$. Here, $T_{i}(i=0, \ldots, 8)$ denote the generators of $U(3)$, while $S_{i}$ represents the scalar, $P_{i}$ the pseudoscalar, $V_{i}^{\mu}$ the vector, and $A_{i}^{\mu}$ the axial-vector meson fields, and $A^{\mu}$ is the electromagnetic field. It should be noted that here and below we use the so-called non strange - strange basis in the $(0-8)$ sector, defined as

$$
\varphi_{N}=\frac{1}{\sqrt{3}}\left(\sqrt{2} \varphi_{0}+\varphi_{8}\right), \quad \varphi_{S}=\frac{1}{\sqrt{3}}\left(\varphi_{0}-\sqrt{2} \varphi_{8}\right), \quad \varphi \in\left(S_{i}, P_{i}, V_{i}^{\mu}, A_{i}^{\mu}\right)
$$

which is more suitable for our calculations. Moreover, $H$ and $\Delta$ are constant external fields defined as

$$
\begin{aligned}
H=H_{0} T_{0}+H_{8} T_{8}=\left(\begin{array}{ccc}
\frac{h_{0 N}}{2} & 0 & 0 \\
0 & \frac{h_{0 N}}{2} & 0 \\
0 & 0 & \frac{h_{0 S}}{\sqrt{2}}
\end{array}\right), \\
\Delta=\Delta_{0} T_{0}+\Delta_{8} T_{8}=\left(\begin{array}{ccc}
\frac{\tilde{\delta}_{N}}{2} & 0 & 0 \\
0 & \frac{\tilde{\delta}_{N}}{2} & 0 \\
0 & 0 & \frac{\tilde{\delta}_{S}}{\sqrt{2}}
\end{array}\right) \equiv\left(\begin{array}{ccc}
\delta_{N} & 0 & 0 \\
0 & \delta_{N} & 0 \\
0 & 0 & \delta_{S}
\end{array}\right) .
\end{aligned}
$$

Throughout this work we assume exact isospin symmetry for $u$ and $d$ quarks, such that the first two diagonal elements in Eqs. (3) and (4) are identical.

All fields in our model represent $\bar{q} q$ states, as discussed in Ref. [6]. In the non-strange sector, we assign the fields $\vec{\pi}$ and $\eta_{N}$ to the pion and the non-strange part of the $\eta$ and $\eta^{\prime}$ mesons, $\eta_{N} \equiv(\bar{u} u+\bar{d} d) / \sqrt{2}$. The fields $\omega_{N}^{\mu}$ and $\vec{\rho}^{\mu}$ represent the $\omega(782)$ and $\rho(770)$ vector mesons, respectively, and the fields $f_{1 N}^{\mu}$ and $\vec{a}_{1}^{\mu}$ represent the $f_{1}(1285)$ and $a_{1}(1260)$ mesons, respectively. In the strange sector, we assign the $K$ fields to the kaons; the $\eta_{S}$ field is the strange contribution to the physical $\eta$ and $\eta^{\prime}$ fields $\left[\eta_{S} \equiv \bar{s} s\right]$; the $\omega_{S}$, $f_{1 S}, K^{\star}$, and $K_{1}$ fields correspond to the $\phi(1020), f_{1}(1420), K^{\star}(892)$, and $K_{1}(1270)$ [or $\left.K_{1}(1400)\right]$ mesons, respectively.

Unfortunately, the assignment of the scalar fields is substantially less clear. Experimental data suggest existence of five scalar-isoscalar states below $1.8 \mathrm{GeV}$ : $f_{0}(500)$, $f_{0}(980), f_{0}(1370), f_{0}(1500)$, and $f_{0}(1710)$, all of which could, in principle, be candidates for $f_{0}^{L}$ and $f_{0}^{H}$. Similarly, the isospin triplet $\vec{a}_{0}$ can be assigned to different physical resonances - although, in this case, there are only two candidate states: $a_{0}(980)$ and $a_{0}(1450)$. An analogous statement holds for the scalar kaon $K_{0}^{\star}$ that can be assigned to the resonances $K_{0}^{\star}(800)$ or $K_{0}^{\star}(1430)$.

In the Lagrangian (1) there are two terms which break the original $U(3)_{L} \times U(3)_{R}$ $\left[=U(3)_{A} \times U(3)_{V}\right]$ symmetry, namely the determinant term, which breaks the $U(1)_{A}$ symmetry, and the explicit symmetry breaking terms of Eqs. (3) and (4), which break $U(3)_{A}$, if $H_{0}, \Delta_{0} \neq 0$ and $U(3)_{V} \rightarrow S U(2)_{V} \times U(1)_{V}$, if in addition $H_{8}, \Delta_{8} \neq 0$ [for more details see, e.g. Ref. [7]]. Besides this explicit symmetry breaking the chiral symmetry

\footnotetext{
${ }^{1}$ With the exception of the $(0-8)$ sector where particle mixing takes place.
} 
is also broken spontaneously. If isospin symmetry is exact, only the $\sigma_{N}$ and $\sigma_{S}$ scalar fields, carrying the same quantum numbers as the vacuum, can have nonzero vacuum expectation values (vev's) ${ }^{2}$. Moreover, the parameter $c_{1}$ describes the axial anomaly, i.e., the $U(1)_{A}$ symmetry is explicitly broken by this term.

After spontaneous symmetry breaking, the fields with nonzero vev's are shifted by their expectation values, namely, $\sigma_{N / S} \rightarrow \sigma_{N / S}+\phi_{N / S}$, where we have introduced $\phi_{N / S} \equiv$ $\left\langle\sigma_{N / S}\right\rangle$. After substituting the shifted fields into the Lagrangian (1), one obtains the treelevel masses by selecting all terms quadratic in the fields. The (square) mass matrices are in general non-diagonal due to the mixing among particles sitting in the center of a given nonet. Besides the mixing inside the nonets there are other terms which mix different nonets because of the vacuum expectation values of the $\sigma$ fields. The mass matrices can be diagonalized by appropriate orthogonal transformations. In order to retain the canonical normalization for the fields, one has to introduce wavefunction renormalization constants, too.

From the Lagrangian one can also derive several decay widths. The mass and decaywidth formulas can be found in Ref. [5].

\section{RESULTS}

We perform a global fit of the parameters of the Lagrangian (1) to experimentally known quantities (decay constants, masses, and decay widths as well as amplitudes), for details see Ref. [5]. We do not include the scalar-isoscalar states into the fit, but we test a posteriori their assignment and phenomenology. We do, however, include the isotriplet and isodoublet quark-antiquark scalar states and we test all four combinations $a_{0}(1450) / K_{0}^{\star}(1430), a_{0}(980) / K_{0}^{\star}(800), a_{0}(980) / K_{0}^{\star}(1430)$, and $a_{0}(1450) / K_{0}^{\star}(800)$. Quite remarkably, the outcome of the fit is univocal: only the pair $a_{0}(1450) / K_{0}^{\star}(1430)$ yields a good fit, while the other combinations do not. We thus conclude that the $I=1$ and $I=1 / 2$ quark-antiquark scalar resonances lie above $1 \mathrm{GeV}$. In fact, the quality of our fit is surprisingly good. As one can see in Fig. 1 we describe all experimental quantities with an average error of 5\%, and most of them even to much better precision.

We then study the two isoscalar mesons of the model. Setting the (large- $N_{c}$ suppressed) parameters $\lambda_{1}$ and $h_{1}$ in the Lagrangian (1) to zero, these masses turn out to be about $1.36 \mathrm{GeV}$ and $1.53 \mathrm{GeV}$, respectively. Our results for $f_{0}^{L}$ are in agreement with the experimental decay widths of $f_{0}(1370)$. Our theoretical value for $f_{0}^{H} \rightarrow K K$ turns out to be too large, while $f_{0}^{H} \rightarrow \pi \pi$ vanishes, because $f_{0}^{H} \equiv \sigma_{S}$ is a pure $\bar{s} s$ state. Nevertheless, our model predicts the existence of a scalar-isoscalar state which decays predominantly into kaons; this is indeed the decay pattern shown by $f_{0}(1710)$. For these reasons we identify our state $f_{0}^{H}$ as (predominantly) $f_{0}(1710)$. It should be stressed that a quantitative study of the scalar-isoscalar system cannot be performed at present because our model does not contain the glueball state, the mass of which is around 1.5 $\mathrm{GeV}$ and which mixes with the two $\bar{q} q$ scalar-isoscalar states, forming the three physical

${ }^{2}$ In case of isospin breaking, also $\sigma_{3}$ would have a nonzero vev. 

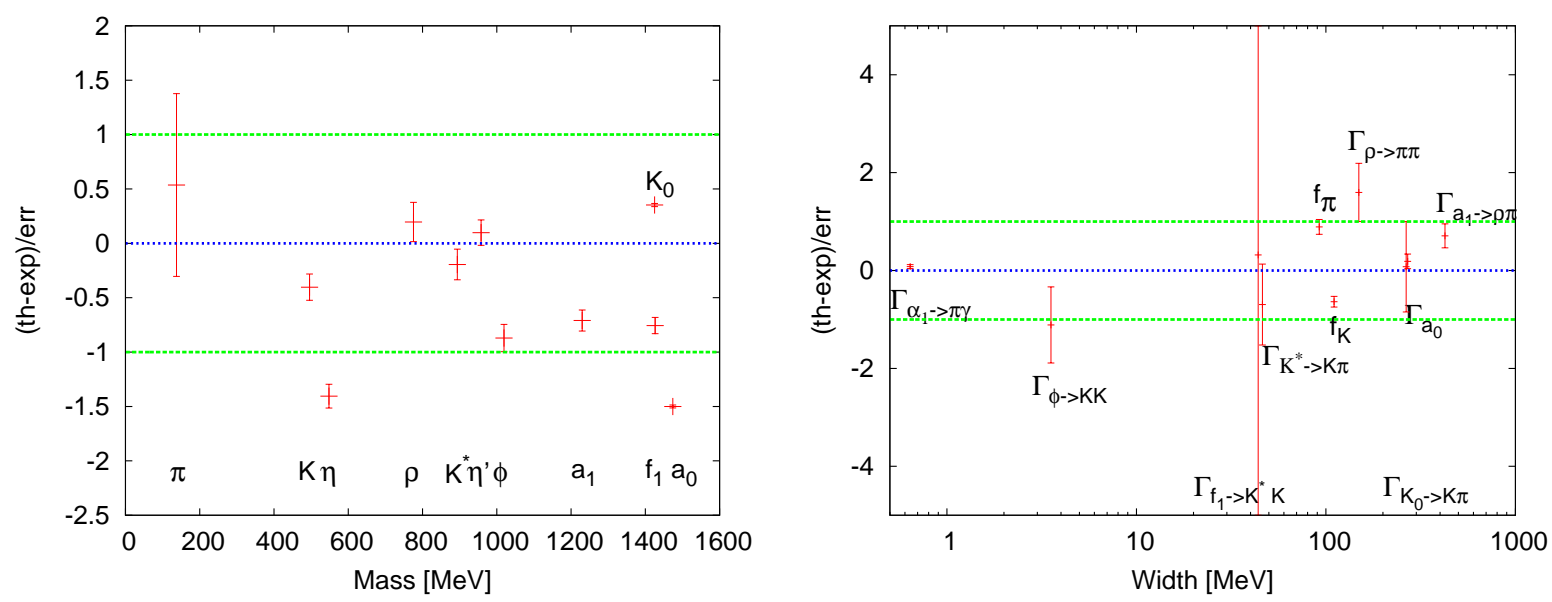

FIGURE 1. Fits to masses and decay widths. Shown is the difference of the theoretical and experimental values divided by the experimental errors. The errorbars correspond to the theoretical errors as emerging from our fit.

states in the $1.3-1.7 \mathrm{GeV}$ energy region.

\section{CONCLUSIONS}

We have presented a linear sigma model with three flavors and global chiral $U(3)_{L} \times$ $U(3)_{R}$ symmetry. The model implements the symmetries of QCD, the discrete $C P T$ symmetry, the global $U\left(N_{f}\right)_{L} \times U\left(N_{f}\right)_{R}$ chiral symmetry, and the breaking mechanisms of the latter symmetry.

The model includes meson states up to energies of $\sim 1.7 \mathrm{GeV}$. This energy region exhibits numerous resonances, related by scattering reactions and decays. For this reason, a realistic model of QCD degrees of freedom in the mentioned energy region should describe as many of the resonances as possible. Thus, we have constructed a linear sigma model that contains scalar (two isoscalars, $f_{0}^{L}$ and $f_{0}^{H}$, as well as an isotriplet, $\vec{a}_{0}$, and two isodoublets, $\left.K_{0}^{\star}\right)$, pseudoscalar $\left(\pi, K, \eta, \eta^{\prime}\right)$, vector $\left(\rho, \omega, K^{\star}, \phi\right)$, and axial-vector $\left[a_{1}, K_{1}, f_{1}(1285), f_{1}(1420)\right]$ degrees of freedom.

The model, dubbed extended Linear Sigma Model (eLSM), has allowed us to study the overall phenomenology of mesons and, in particular, to explore the nature of scalar and axial-vector resonances. In order to test our model we have performed a global fit to 21 experimental quantities involving both the (pseudo)scalar and the (axial-)vector masses and decays. Due to mixing with the scalar glueball (not included here), we did not include the scalar-isoscalar resonances in the fit. Similarly, we have omitted the axial-vector resonance $K_{1}$, due to the fact that in reality a large mixing of two kaonic fields from the $1^{++}$and $1^{+-}$nonets takes place.

One of the central questions of our discussions has been the assignment of the scalar states: to this end we have tested the possible scenarios for the isotriplet and isodoublet scalar states by assigning our scalar fields $\vec{a}_{0}$ to $a_{0}(980)$ or $a_{0}(1450)$ and $K_{0}^{\star}$ to $K_{0}^{\star}(800)$ or $K_{0}^{\star}(1430)$. The outcome is univocal: the global fit works well only if the states 
$a_{0}(1450)$ and $K_{0}^{\star}(1430)$ are interpreted as quark-antiquark states. On the contrary, the other combinations deliver large and unacceptable values of $\chi^{2}$. We thus conclude that the scalar $I=1$ and $I=1 / 2$ states have to be identified with the resonances $a_{0}(1450)$ and $K_{0}^{\star}(1430)$. Moreover, the overall phenomenology described by the fit is very good, see Ref. [5]. The good agreement with data also shows that the axial-vector mesons can be interpreted, just as their vector chiral partners, as quark-antiquark states.

We have then studied the consequences of our fit. We have primarily concentrated on the scalar-isoscalar sector which was not included in the fit. In the large $N_{c}$ limit it is possible to make clear predictions for the two states $f_{0}^{L}$ and $f_{0}^{H}$. Their masses lie above $1 \mathrm{GeV}$ and their decay patterns have led us to identify $f_{0}^{L}$ with (predominantly) $f_{0}(1370)$ and $f_{0}^{H}$ with (predominantly) $f_{0}(1710)$. The theoretical decay rates of $f_{0}^{L}$ are in agreement with experiment; $f_{0}^{H}$ decays only into kaons, but turns out to be too wide. At a qualitative level, this large- $N_{c}$ result clearly shows that also the scalar-isoscalar quarkantiquark states lie above $1 \mathrm{GeV}$. However, in this system the inclusion of an additional glueball state would be necessary for a full quantitative study. Mixing phenomena are known to be large here and affect both the masses and decays. The study of a genuine three-state system is mandatory: starting from $\sigma_{N}=\sqrt{1 / 2}(\bar{u} u+\bar{d} d), G=g g$ and $\sigma_{S}=\bar{s} s$ to describe properly $f_{0}(1370), f_{0}(1500)$, and $f_{0}(1710)$.

\section{ACKNOWLEDGMENTS}

Gy. Wolf and P. Kovács thank Goethe University for its hospitality. They were partially supported by the Hungarian OTKA funds T71989 and T101438. The work of D. Parganlija and F./ Giacosa was supported by the Foundation of the Polytechnical Society Frankfurt. This work was financially supported by the Helmholtz International Center for FAIR within the framework of the LOEWE program (Landesoffensive zur Entwicklung Wisschenschaftlich-Ökonomischer Exzellenz) launched by the State of Hesse.

\section{REFERENCES}

1. J. Beringer et al. (Particle Data Group), Phys. Rev. D 86, 010001 (2012).

2. A. Heinz, S. Struber, F. Giacosa and D. H. Rischke, Phys. Rev. D 79, 037502 (2009) [arXiv:0805.1134 [hep-ph]]; A. Heinz, S. Struber, F. Giacosa and D. H. Rischke, Acta Phys. Polon. Supp. 3, 925 (2010) [arXiv:1006.5393 [hep-ph]].

3. J. S. Schwinger, Annals Phys. 2, 407 (1957); M. Gell-Mann and M. Levy, Nuovo Cim. 16, 705 (1960); S. Weinberg, Phys. Rev. Lett. 18, 188 (1967).

4. J. S. Schwinger, Phys. Lett. B 24, 473 (1967); S. Weinberg, Phys. Rev. 166, 1568 (1968).

5. D. Parganlija, P. Kovács, Gy. Wolf, F. Giacosa and D. H. Rischke, [arXiv:1208.0585 [hep-ph]]

6. D. Parganlija, F. Giacosa and D. H. Rischke, Phys. Rev. D 82, 054024 (2010) [arXiv:1003.4934 [hep$\mathrm{ph}]]$.

7. J. T. Lenaghan, D. H. Rischke and J. Schaffner-Bielich, Phys. Rev. D 62, 085008 (2000) [nuclth/0004006]; 